\title{
Custom-Made Mouthguards: Electromyographic Analysis of Masticatory Muscles and Cardiopulmonary Tests in Athletes of Different Sports
}

\author{
D. Tripodi, D. Fulco, A. Beraldi, P. Ripari, G. Izzi, S. D'Ercole* \\ Department of Medical, Oral and Biotechnological Sciences, University “G. d’Annunzio” of Chieti-Pescara, Chieti, Italy \\ Email: *simonettadercole@gmail.com
}

How to cite this paper: Tripodi, D., Fulco, D., Beraldi, A., Ripari, P., Izzi, G. and D'Ercole, S. (2019) Custom-Made Mouthguards: Electromyographic Analysis of Masticatory Muscles and Cardiopulmonary Tests in Athletes of Different Sports. Health, 11, 428-438.

https://doi.org/10.4236/health.2019.114038

Received: February 27, 2019

Accepted: April 27, 2019

Published: April 30, 2019

Copyright $\odot 2019$ by author(s) and Scientific Research Publishing Inc. This work is licensed under the Creative Commons Attribution International License (CC BY 4.0).

http://creativecommons.org/licenses/by/4.0/

\begin{abstract}
The purpose of this study is to analyse the neuromuscular balance of the masticatory muscles and the influence on the athlete respiratory parameters induced by custom-made mouthguard. Twenty-six athletes ( 24 males and $2 \mathrm{fe}$ males), of different disciplines, average age of $32.12 \pm 12.05$, were recruited. Each athlete received a custom-made mouthguard in the Ethylene-Vinyl-Acetate (EVA) thermoplastic material and using surface electromyography, the masseter muscles and the frontal beams of the temporal muscles were analysed, with and without mouthguard. The athletes were then subjected to a medical examination and cardiopulmonary test, in the condition with and without mouthguard. The use of mouthguard caused an improvement of all the electromyographic indexes analysed. In particular, GLOBAL INDEX $(p=0.0021), \operatorname{BAR}(\mathrm{p}=0.0005)$, IMPACT $(\mathrm{p}=0.0076)$ and ASIM ( $p=0.0290)$ showed an enhancement statistically significant. For the cardiopulmonary test indexes as maximum oxygen uptake $\left(\mathrm{VO}_{2} \mathrm{max}\right)$, minute ventilation (VE), breathing reserve (BR), not statistically differences ( $\mathrm{p}>$ 0.05 ) were reported. The custom-made mouthguard improved the neuromuscular balance of the masticatory muscles, symmetrizing the masseters and temporalis muscles work. It produced a better balancing distribution of occlusal loads both in the anterior-posterior direction and in the lateral direction and offered the possibility to produce more muscular work. Moreover, it did not constitute an impediment that can significantly disturb the athlete's breathing during physical activity.
\end{abstract}

\section{Keywords}

Mouthguard, Sport, Masticatory Muscles, Neuromuscular Balance, 
Electromyography

\section{Introduction}

One of the most interesting aspects of modern dentistry is the evaluation of the correlations between the stomatognathic system and body posture. In the last decade, the relationship between postural imbalance, malocclusion and craniomandibular disorders has been the object of great interest in the oral dentistry.

This interest was however favoured by the great social impact resulting from the increase in information, linked to the disclosure of these issues by the mass media. The relationship between occlusion and posture in the performance of the sporting exercise has been discussed for a long time. Only in recent years, several authors reported about the malocclusion and the correlation with the athlete's posture, and their effect on performance and on increasing risk of injury [1]. In competitive athletes even a slight change in balance can be translated into variations in the intensity of strength and coordination skills, as well as causing the onset of a state of muscular tensions that negatively affect the whole body, diminishing its athletic potential global.

The lack of occlusal stability could create an energy escape point similar to the sliding of the athlete's foot and an imbalance between the agonist and antagonist muscles, connected together in synergistic chains, which could be the cause of accidents and repeated muscle tears [2].

Sport mouthguards were dental devices that have been utilized by athletes who recognized the need for oral protection during their sports activities [3] [4]. Moreover they allow a stable muscle activity during the training of Karate-Dō athletes [5].

During sport training, the muscles of the cranio-cervico-mandibular complex were recruited, but regards to the effects of a custom-made mouthguard on sport performance, the results in literature are still discordant [6] [7] [8]. The reason for this discrepancy lies in the inter-individual variability that assumed a fundamental role. Therefore, each athlete must be assessed individually with appropriate clinical and instrumental analysis. D'Ercole et al. demonstrated that the use of fixed and/or removable dental devices can affect the oral cavity homeostasis [9]. Indeed, the training time was the period in which there was a massive growth of intraoral bacteria, accompanied by a statistically significant decrease in the concentration of S-IgA. Therefore it is necessary to monitor the athlete's oral environmental changes to reduce the risk of oral disease during a sport treatment [10] [11].

Electromyographic analysis and cardiopulmonary tests were chosen for this work, in order to analyse the chewing muscles in athletes who used individual mouthguards during sport activities. The aim of this study was to evaluate any variations on the neuromuscular balance induced by the mouthguard and to 
compare it to the athlete dental occlusion in maximum intercuspidation. The study also analysed the influence of the custom-made mouthguard on the athlete respiratory parameters under physical effort.

\section{Materials and Methods}

The study was conducted at the Department of Medical, Oral and Biotechnological Sciences of the University of "G. d'Annunzio" Chieti-Pescara, in the period 2017-18. Electromyographic analysis and dental examinations were carried out in the Dental Operative Unit, while specific medical examinations and cardiopulmonary tests were performed in the Operative Unit of Medicine and Sport Cardiology. All the mouthguards were made in the Dental laboratory of Dental School.

\subsection{Study Population}

The study population was composed of 26 subjects ( 24 males and 2 females): 4 triathlon athletes, 6 volleyball players, 5 skiers, 9 hockey players, 1 squash player and 1 kick boxer. They showed the following characteristics: average age $32.12 \pm$ 12.05 , average weight $80.30 \pm 9.73 \mathrm{~kg}$ and average height $184.30 \pm 10.90 \mathrm{~cm}$.

The following inclusion criteria were considered: general good health, good dental health, dental care not in progress, need for sport treatment with mouthguards. The following exclusion criteria were considered: acute or chronic conditions that could limit the ability of the athlete to participate in the study; failure to use the mouthguard.

The selected subjects participated voluntarily in the study. Patients were first given oral and written information on the study's purpose. Informed consent was given by signing a protocol (Privacy Law DL 196/2003). In this study, the approval from the Ethical Committee it's not reported because that's not required for works that are based on research protocol on medical devices already used in the clinical protocols approved from the Department for the medical use.

A self-administered questionnaire was used to obtain data concerning a pathological complete history, a history of hard and soft tissues of the oral cavity, a family history, oral hygiene practices.

\subsection{Clinical Monitoring}

A clinical monitoring was performed and on each patient were recorded the number of decayed (D), missing (M) and filled (F) teeth (T) (DMFT) to assess caries prevalence according to WHO criteria. To evaluate oral hygiene and periodontal status, the Plaque Index (PL+) according to Silness $\&$ Löe, and the Löe \& SilnessGingival Bleeding (BOP+) were used, respectively [12].

In addition, an oral examination of intraoral mucosal was performed and assessed the presence/absence of bad habits and/or parafunctional habits.

Custom mouthguards were made in a dental laboratory on models cast from impressions and using Ethylene-Vinyl-Acetate (EVA) materials. 
For each athlete a silicone impression was take off and the occlusal waxes, using extra-hard "Beauty Pink" wax (Integra ${ }^{\mathrm{TM}}$ Miltex $^{\circledast}$ ) combined with a soft "tenatex" wax (Howmedica-kemdent ${ }^{\circledR}$ ), was registered. These registration allowed the production of individual mouthguards.

Preliminary correction and adjustment in static and dynamic occlusion were performed for each newly fabricated mouthguard. Finally, each player was instructed on oral and mouthguard hygiene.

Each subject has repeated the indications received so the clinician was able to correct any inaccuracy.

Analysis of the masseter muscles and the anterior beams of the temporal muscles were carried out on all subjects by surface electromyography, with and without mouthguard.

The 26 athletes were also subjected to a medical examination and cardiopulmonary test, using an Ergoselect 100 ergometer (Ergoline ${ }^{\mathrm{rm}}$ Bad Honnef) an Ergostick ergospirometer (Geratherm Respiratory GmbH, Bad Kissingen) and an “Amedtec EcgPro" computerized stress electrocardiograph (AMEDTEC Medizintechnik Aue GmbH, Aue).

Each athlete performed a first test without the mouthguard and after at least 48 hours, a second test wearing the mouthguard.

\subsection{Mouthguard Manufacturing}

Custom-made mouthguards were created in the EVA thermoplastic material. After the dentist took impressions and registered occlusions with wax, the dental technician proceeded to develop the plaster models of the patient's dental arches and to perform the assembly in the articulator, to ensure maximum precision.

The mouthguards were made using "Playsafe" thermoforming discs (ERKODENT ${ }^{\circledast}$ Erich Kopp GmbH, Pfalzgrafenweiler), with a diameter of 120 $\mathrm{mm}$ and a thickness of $5.5 \mathrm{~mm}$ triple layer, using a thermoforming technique with the "Erkoform-3d" machine (ERKODENT ${ }^{\circledast}$ Erich Kopp GmbH, Pfalzgrafenweiler). To ensure comfort and protection, the criteria indicated by Patrick et al. was followed [13].

\subsection{Electromyography}

The "BTS TMJOINT ${ }^{\mathrm{Tm}}$ " (BTS Bioengineering Corp., Brooklyn) was used as electromyograph, together with "Kendall ${ }^{\mathrm{TM}} \mathrm{Arbo}^{\mathrm{Tm}} \mathrm{H} 124 \mathrm{SG}$ " surface EMG electrodes (Covidien, Dublin). Bipolar electrodes captured the action potentials from the muscles studied, and the surface electrodes were positioned parallel to the muscle fibres. Their position is obligatory with numerical code of the electrodes: 1 right temporal, 2 left temporal, 3 right masseter, 4 left masseter (Figure 1).

The clenching test was performed on the cotton rolls, necessary for standardizing the values: the ratio between basal values (clenching without cotton rollers) and those referring to the frame on the cotton rolls, identified the influence of dental occlusion on neuromuscular balance. The $n^{\circ} 3$ cotton rolls were posi- 
tioned parallel to the dental arch, on the occlusal surface behind the first premolars, in order to avoid dental contact. After the clenching tests performed on the cotton rolls and in maximum intercuspation, the procedures previously described were also followed for the custom made mouthguards. The tests were performed in the same way and each for the duration of $5 \mathrm{sec}$, and the athletes were invited to clench as hard as possible, and to maintain the same level of contraction for all the recording. During tests performance, the atlhetes were verbally encouraged to perform at their best.

In the electromyographic analysis of the present study, were used the symmetry indexes proposed by Ferrario et al. and widely used in scientific literature [14] [15].

The indexes considered were the following:

- GLOBAL INDEX: parameter that summarizes the main occlusal indexes;

- POC TA: percentage overlapping coefficient for anterior temporalis;

- POC MM: percentage overlapping coefficient for masseter;

- BAR: index of the occlusal barycentre;

- TORS: mandibular torsion index;

- IMPACT: standardized activity index;

- ASIM: index of asymmetry in absolute value.

These indexes expressed as a percentage as indicated by Ferrario et al. [14].

\subsection{Cardiopulmonary Test Procedure}

The cardiopulmonary test performed in this study was of maximal incremental type, with the ramp method that allowed to increase the work load in a more physiological way. The first fundamental step consisted in the calibration of the instruments, after which the electrodes were applied to the patient for the monitoring of all 12 electrocardiographic derivations and the mask was positioned on the face to convey all the respiratory flows inside the ergospirometer. It was important at this stage to check that there were no air leaks between the mask and the skin and reminded the patient to breathe exclusively with the mouth (Figure 2).

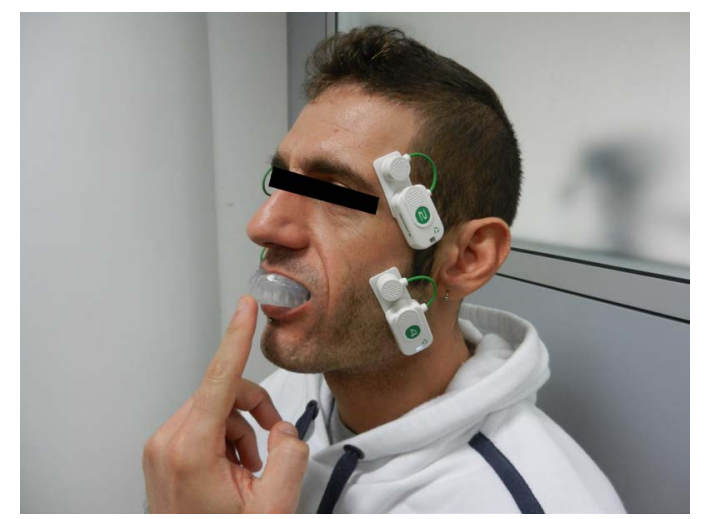

Figure 1. The athlete during electromyography analysis with mouthguard: the surface electrodes were positioned parallel to the muscle fibres of masseters and temporalis. 


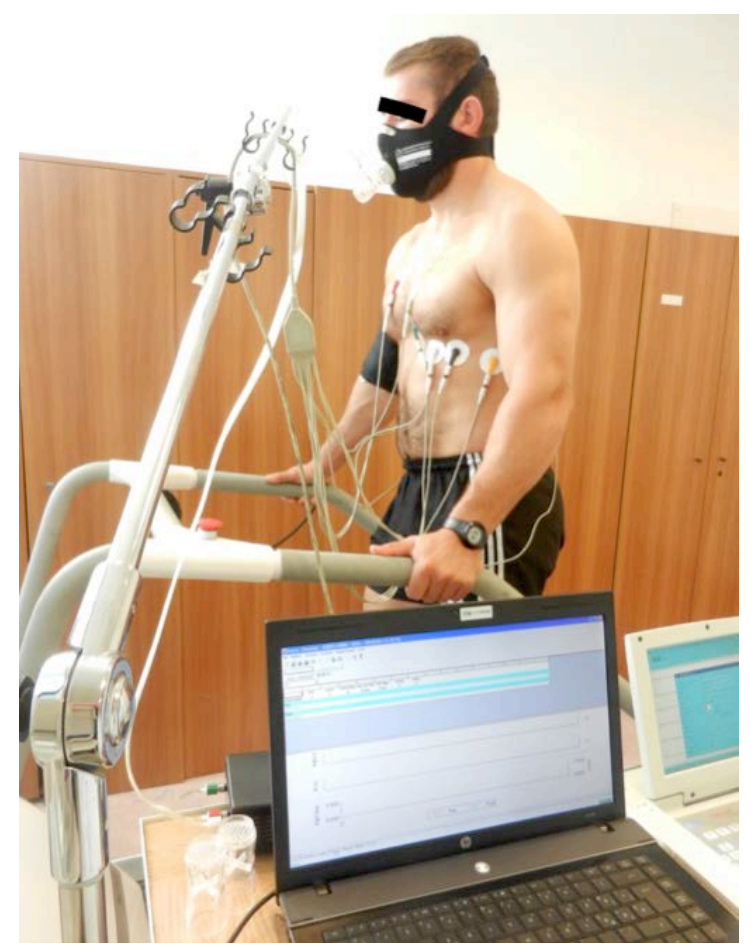

Figure 2. The athlete during cardiopulmonary test.

At this point, the athlete was positioned on the mobile platform, and basal values acquired. The test began with a preheating with workloads of lower intensity (25 Watts). During the stress test, the number of cycles per min to maintain increased (70 - 80 cycles per $\mathrm{min}$ ) and the loads were greater (increases of 70 Watts every $2 \mathrm{~min}$ ), until the maximum tolerability of the individual was reached, or rather, when they were no longer able to continue running. Generally, the incremental test lasted between 8 and $10 \mathrm{~min}$, while the next phase of recovery (with the subject at rest) occured in a variable time between 2 and $5 \mathrm{~min}$. The data of the cardiopulmonary parameters acquired and monitored graphically in real time throughout the test, were finally processed by software.

Environmental conditions necessary:

- temperature between $18^{\circ} \mathrm{C}-22^{\circ} \mathrm{C}$;

- relative humidity not higher than $55 \%$;

- well-ventilated environment;

- calm and silence around the subject.

The parameters analyzed by the cardiopulmonary test are:

- maximum oxygen uptake $\left(\mathrm{VO}_{2} \max \right)$;

- minute ventilation (VE);

- breathing reserve (BR).

\subsection{Statistic Analysis}

The data are expressed as mean values \pm standard deviation. Statistical significance was accepted at $\mathrm{P}<0.05$. 


\section{Results}

Data collected from dental examination on athletes are show in Figure 3. The athletes demonstrated a very poor oral health, as confirmed by high values of clinical indexes: DMFT $7 \pm 4.45, \mathrm{PL}+41.18 \%$ and BOP $+29.41 \%$. Athletes with a previous history of dental trauma were $52.94 \%$, but only the $23.53 \%$ of players used boil \& bite mouthguard.

$29.41 \%$ of athletes reported temporomandibular joint disorders, $37.50 \%$ history of orthodontic appliances, $50 \%$ presence of oral habits and $23.08 \%$ were affected by bruxism (Figure 3).

Table 1 shows the results collected by electromyographic analysis on the 26 athletes analysed in the present study.

By comparing the mean values ("A" and " $\mathrm{B}$ ") for all the considered indexes, can be observed that there was an improvement in the (B) condition with mouthguard. In particular improvements statistically significant were registered in the following indexes: the GLOBAL INDEX (85.15 \pm 5.55 without mouthguard, $88.92 \pm 1.02$ with mouthguard, $\mathrm{p}<0.05)$; the BAR $(82.79 \pm 9.34$, for $\mathrm{A}$; $89.75 \pm 2.12$ for $\mathrm{B}, \mathrm{p}<0.05)$; the IMPACT $(99.12 \pm 49.02$ for $\mathrm{A}, 126.35 \pm 46.40$ for $\mathrm{B}, \mathrm{p}<0.05)$; the ASIM (5.67 \pm 4.52 for $\mathrm{A}, 3.93 \pm 2.59$ for $\mathrm{B}, \mathrm{p}<0.05)$.

Table 2 shows the results relating to respiratory parameters, obtained from the cardiopulmonary test. By analysing the results, can be observed slight differences between the condition " $\mathrm{A}$ " (without mouthguard) and condition " $\mathrm{B}$ " (with mouthguard).

Table 1. Electromyographic results revealed in athletes in different conditions: without mouthguard (A) and with mouthguard (B).

\begin{tabular}{ccc}
\hline & A & B \\
\hline Global Index & $85.15 \pm 5.55$ & $88.92 \pm 1.02^{\S}$ \\
POC TA & $87.12 \pm 2.47$ & $87.75 \pm 1.35$ \\
POC MM & $83.12 \pm 9.65$ & $86.31 \pm 2.06$ \\
BAR & $82.79 \pm 9.34$ & $89.75 \pm 2.12^{\S}$ \\
TORS & $89.75 \pm 4.54$ & $90.98 \pm 1.05$ \\
IMPACT & $99.12 \pm 49.02$ & $126.35 \pm 46.40^{\S}$ \\
ASIM & $5.67 \pm 4.52$ & $3.93 \pm 2.59^{\S}$ \\
\hline
\end{tabular}

$\S \mathrm{Sp}<0.05$.

Table 2. Respiratory parameters detected in athletes in conditions without mouthguard (A) and with mouthguard (B).

\begin{tabular}{ccc}
\hline & A & B \\
\hline VO $_{2} \max$ & $50.35 \pm 11.47$ & $49.15 \pm 11.52$ \\
VE & $119.75 \pm 22.10$ & $122.25 \pm 27.65$ \\
BR & $18.25 \pm 12.23$ & $17.50 \pm 14.71$ \\
\hline
\end{tabular}




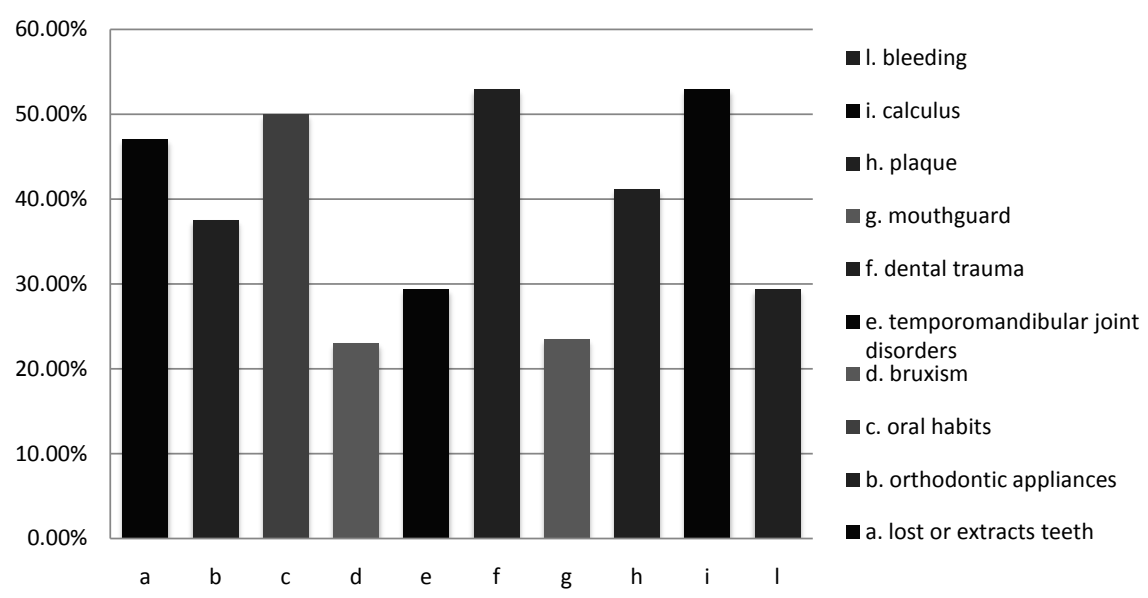

Figure 3. Prevalence (\%) of clinical characteristics of the studied populations.

\section{Discussion}

The present study demonstrated that a correctly performed individual mouthguard, in addition to trauma prevention, offered a better neuromuscular balance of the masticatory muscles.

The electromyography analysis showed that an improvement occured for several analysed indexes, in the condition with mouthguard. In particular, four parameters significantly improved: GLOBAL INDEX, BAR, IMPACT and ASIM. This involved that individual mouthguard symmetrized the work of masseter and temporal muscles to the point of better balancing the distribution of occlusal loads both in the anterior-posterior direction and in the lateral direction and offered the possibility of producing more muscular work. Moreover, it also resulted in a better comfort for the athlete, as well as a series of possible improvements on the activation of muscle chains, with positive repercussions on posture, balance and inevitably, on athletic performance.

Raquel et al. [5] described a study concerning the electromyographic analysis of masticatory muscles in subjects who used individual mouthguards.

The authors compared the electromyographic activity of the masticatory muscles before and after training, with and without the use of a mouthguard by different indexes in respect to present study.

They observed that the electromyographic parameters remained unchanged; in this way, stable muscle activity was allowed during training [16]. These results corroborated the hypothesis of the present study: the custom made mouthguard must be well calibrated and not altered the masticatory muscle activity. The muscle activity must be stable or as reported in this study, improved.

Moreover, in the present study the athletes wearing an individual mouthguard were performed by means of cardiopulmonary test.

The results highlighted slight differences between the condition without and with mouthguard.

These data encouraged the use of the individual mouthguard because it should not interfere with the athlete's respiratory function. 
These results confirmed what is reported in the literature [17] [18]: wearing a custom made mouthguard well calibrated during sporting activities did not alter the athlete's main respiratory physiological parameters. These results provided further support to encouraging athletes to wear the mouthguard during sport activities.

The individual mouthguards were distinguished by their ability to offer the patient adequate protection, comfort in use and adequate aesthetics given by soft tissue support, as well as the possibility of customizing the device.

The relationship between mouthguard and ecological changes in the oral cavity is still under debate. Instead of this, various strategies can be taken to avoid ecological changes in the oral cavity due to use of mouthguards, and different substances can be applied inside the mouthguard, e.g. chlorhexidine which is commonly used as an active ingredient to prevent the formation of plaque and to reduce its pathogenicity [19].

Moreover, the application of casein, within custom-made EVA mouthguards, positively influenced salivary flow, increased $\mathrm{pH}$ values, the amount of stimulated saliva and the buffering capacity of the athlete, improving their state of oral health, which was negatively affected by the use of common mouthguards [20].

The present study demonstrated that electromyographic analysis, cardiopulmonary test associated with previously reported tests as microbial markers, immune status and sporting characteristics [21] were important for establishing guidelines for management of training load in order to minimize physical stress and the risk of oral infection.

The lack of literature highlights the need for further study on a very current problem in the field of sport dentistry.

\section{Conclusion}

The individual mouthguard significantly improved the neuromuscular balance of the masticatory muscles, symmetrizing the work of the masseter and temporal muscles to the point of better balancing the distribution of occlusal loads both in the anterior-posterior direction and in the lateral direction and offered the possibility of producing more muscular work. Moreover, it did not constitute an impediment that significantly disturbs the athlete's breathing during physical exertion.

\section{Declaration of Conflicting Interests}

The authors declared no potential conflicts of interest with respect to the research, authorship, and/or publication of this article.

\section{References}

[1] Schultz Martins, R., Girouard, P., Elliott, E. and Mekary, S. (2018) Physiological Responses of a Jaw-Repositioning Custom-Made Mouthguard on Airway and Their Effects on Athletic Performance. The Journal of Strength and Conditioning Research, 1-7. https://doi.org/10.1519/JSC.0000000000002679 
[2] Julià-Sánchez, S., Álvarez-Herms, J. and Burtscher, M. (2019) Dental Occlusion and Body Balance: A Question of Environmental Constraints? Journal of Oral Rehabilitation, 46, 388-397. https://doi.org/10.1111/joor.12767

[3] Knapik, J.J., Marshall, S.W., Lee, R.B., Darakjy, S.S., Jones, S.B., Mitchener, T.A., DelaCruz, G.G. and Jones, B.H. (2007) Mouthguards in Sport Activities: History, Physical Properties and Injury Prevention Effectiveness. Sports Medicine, 37, 117-144. https://doi.org/10.2165/00007256-200737020-00003

[4] Glass, R.T., Conrad, R.S., Wood, C.R., Warren, A.J., Kohler, G.A., Bullard, J.W., Benson, G. and Gulden, J.M. (2019) Protective Athletic Mouthguards: Do They Cause Harm? Sports Health, 1, 411-415. https://doi.org/10.1177/1941738109341441

[5] Raquel, G., Namba, E.L., Bonotto, D., Ribeiro Rosa, E.A., Trevilatto, P.C., Naval Machado, M.Â., Vianna-Lara, M.S. and Azevedo-Alanis, L.R. (2017) The Use of a Custom-Made Mouthguard Stabilizes the Electromyographic Activity of the Masticatory Muscles among Karate-Dō Athletes. Journal of Bodywork and Movement Therapies, 21, 109-116. https://doi.org/10.1016/j.jbmt.2016.05.007

[6] Valentino, B., Fabozzo, A. and Melito, F. (1991) The Functional Relationship between the Occlusal Plane and the Plantar Arches. An EMG Study. Surgical and Radiologic Anatomy, 13, 171-174. https://doi.org/10.1007/BF01627980

[7] Bracco, P., Deregibus, A. and Piscetta, R. (2004) Effects of Different Jaw Relations on Postural Stability in Human Subjects. Neuroscience Letters, 356, 228-230. https://doi.org/10.1016/j.neulet.2003.11.055

[8] Cuccia, A. and Caradonna, C. (2009) The Relationship between the Stomatognathic System and Body Posture. Clinics (Sao Paulo), 64, 61-66. https://doi.org/10.1590/S1807-59322009000100011

[9] D’Ercole, S., Martinelli, D. and Tripodi, D. (2014) Influence of Sport Mouthguards on the Ecological Factors of the Children Oral Cavity. BMC Oral Health, 5, 14-97. https://doi.org/10.1186/1472-6831-14-97

[10] D'Ercole, S., Ristoldo, F., Quaranta, F., Amaddeo, P. and Tripodi, D. (2013) Analysis of Oral Health Status and of Salivary Factors in Young Soccer Players: A Pilot Study. Medicina Dello Sport, 66, 71-80.

[11] D'Ercole, S. and Tripodi, D. (2013) The Effect of Swimming on Oral Ecological Factors. Journal of Biological Regulators \& Homeostatic Agents, 27, 551-558.

[12] World Health Organization (1997) Oral Health Survey Basic Methods.

[13] Patrick, D.G., van Noort, R. and Found, M.S. (2005) Scale of Protection and the Various Types of Sports Mouthguard. British Journal of Sports Medicine, 39, 278-281. https://doi.org/10.1136/bjsm.2004.012658

[14] Ferrario, V.F., Sforza, C., Colombo, A. and Ciusa, V. (2000) An Electromyographic Investigation of Masticatory Muscles Symmetry in Normo-Occlusion Subjects. Journal of Oral Rehabilitation, 27, 33-40. https://doi.org/10.1046/j.1365-2842.2000.00490.x

[15] De Felício, C.M., Sidequersky, F.V., Tartaglia, G.M. and Sforza, C. (2009) Electromyographic Standardized Indices in Healthy Brazilian Young Adults and Data Reproducibility. Journal of Oral Rehabilitation, 36, 577-583.

https://doi.org/10.1111/j.1365-2842.2009.01970.x

[16] Bourdin, M., Brunet-Patru, I., Hager, P.E., et al. (2006) Influence of Maxillary Mouthguards on Physiological Parameters. Medicine \& Science in Sports \& Exercise, 38, 1500-1504. https://doi.org/10.1249/01.mss.0000228952.44850.eb

[17] Kececi, A.D., Cetin, C., Eroglu, E. and Baydar, M.L. (2005) Do Custom-Made 
Mouth Guards Have Negative Effects on Aerobic Performance Capacity of Athletes? Dental Traumatology, 21, 276-280.

https://doi.org/10.1111/j.1600-9657.2005.00354.x

[18] Gebauer, D.P., Williamson, R.A., Wallman, K.E. and Dawson, B.T. (2011) The Effect of Mouthguard Design on Respiratory Function in Athletes. Clinical Journal of Sport Medicine, 21, 95-100. https://doi.org/10.1097/JSM.0b013e31820428b0

[19] D’Ercole, S., Tieri, M., Fulco, D., Martinelli, D. and Tripodi, D. (2017) The Use of Chlorhexidine in Mouthguards. Journal of Biological Regulators and Homeostatic Agents, 31, 487-493.

[20] Tripodi, D., Martinelli, D., Ciaravino, C., Fulco, D., Tieri, M. and D’Ercole, S. (2018) The Use of Casein in Sport Mouthguards: Microbiological and Ecological Variations in Oral Cavity. Journal of Biological Regulators and Homeostatic Agents, 32, 1045-1049.

[21] D’Ercole, S., Tieri, M., Martinelli, D. and Tripodi, D. (2016) The Effect of Swimming on Oral Health Status: Competitive versus Non-Competitive Athletes. Journal of Applied Oral Science, 24, 107-113.

https://doi.org/10.1590/1678-775720150324 